\title{
BMJ Open Protocol for the study of cervical cancer screening technologies in HIV-infected women living in Rwanda
}

\author{
Gad Murenzi, ${ }^{1}$ Jean-Claude Dusingize, ${ }^{1}$ Theogene Rurangwa, ${ }^{1}$ \\ Jean d'Amour Sinayobye, ${ }^{1}$ Athanase Munyaneza, ${ }^{1}$ Anthere Murangwa, ${ }^{1}$ \\ Thierry Zawadi, ${ }^{1}$ Tiffany Hebert, ${ }^{2}$ Pacifique Mugenzi, ${ }^{1}$ Adebola Adedimeji, ${ }^{2}$ \\ Leon Mutesa, ${ }^{1,3}$ Kathryn Anastos, ${ }^{2}$ Philip E Castle ${ }^{2}$
}

To cite: Murenzi G, Dusingize J-C, Rurangwa T, et al. Protocol for the study of cervical cancer screening technologies in HIV-infected women living in Rwanda. BMJ Open 2018;8:e020432. doi:10.1136/ bmjopen-2017-020432

- Prepublication history and additional material for this paper are available online. To view these files, please visit the journal online (http://dx.doi. org/10.1136/bmjopen-2017020432).

Received 4 November 2017 Revised 19 June 2018 Accepted 22 June 2018

\section{Check for updates}

(c) Author(s) (or their employer(s)) 2018. Re-use permitted under CC BY-NC. No commercial re-use. See rights and permissions. Published by BMJ.

${ }^{1}$ Rwanda Military Hospital, Kigali, Rwanda

${ }^{2}$ Albert Einstein College of Medicine, Bronx, New York, USA ${ }^{3}$ University of Rwanda, Kigali, Rwanda

Correspondence to

Dr Gad Murenzi;

gadcollins@gmail.com

\section{ABSTRACT}

Introduction The optimal method(s) for screening HIVinfected women, especially for those living in sub-Saharan Africa, for cervical precancer and early cancer has yet to be established.

\section{Methods and analysis A convenience sample of}

$>5000$ Rwandan women, ages 30-54 years and living with HIV infection, is being consented and enroled into a crosssectional study of cervical cancer screening strategies. Participants are completing an administered short risk factor questionnaire and being screened for high-risk human papillomavirus (hrHPV) using the Xpert HPV assay (Cepheid, Sunnyvale, California, USA), unaided visual inspection after acetic acid (VIA) and aided VIA using the Enhanced Visual Assessment (EVA) system (Mobile ODT, Tel Aviv, (srael). Women positive for hrHPV and/or by unaided VIA undergo colposcopy, which includes the collection of two cervical specimens prior to undergoing a fourquadrant microbiopsy protocol. The colposcopy-collected specimens are being tested by dual immunocytochemical staining for p16 $6^{\text {INK4a }}$ and Ki-67 (CINtec PLUS Cytology, Ventana, Tucson, Arizona, USA) and for E6 or E7 oncoprotein for 8 hrHPV genotypes (HPV16, 18, 31, 33, $35,45,52$ and 58) using the next-generation AV Avantage hrHPV E6/E7 test (Arbor Vita Corporation, Freemont, California, USA). Women with a local pathology diagnosis of cervical intraepithelial neoplasia grade 2 (CIN2) or more severe (CIN2+) or pathology review diagnosis of CIN grade three or more severe (CIN3+) will receive treatment. Clinical performance and cost-effectiveness (eg, sensitivity, specificity and predictive values) of different screening strategies and algorithms will be evaluated.

Ethics and dissemination The protocol was approved by local and institutional review boards for human subjects research. At the completion of the study, results will be disseminated to the scientific community through peerreviewed publication and to the Rwandan stakeholders through an external advisory panel.

\section{INTRODUCTION}

Invasive cervical cancer (ICC) remains a significant cause of morbidity and mortality globally. Approximately 530000 cases of and 270000 deaths due to ICC occur annually,

\section{Strengths and limitations of this study}

- We are enroling a very large sample size of HIVinfected women living in Rwanda who otherwise would probably not get cervical cancer screening

- We are employing rigorous disease ascertainment protocols to minimise misclassification.

- Some tests, such as the Xpert HPV and the AV Avantage HPV E6/E7 assays, are being done on site in Rwanda using technologies that could feasibly be deployed there.

- A weakness of the study is that cervical cytology is not being included as a comparator due to financial and logistical constraints.

making it the fourth most common malignancy and cause of cancer-related death in women globally. ${ }^{1}$ In many high-income countries, ICC rates have declined by $50 \%$ or more $^{2}$ due to the introduction of effective, high-coverage Pap testing (cervical cytology)-based screening programmes that include timely follow-up of screen positives, treatment of women with precursor lesions and management of cancers. Now, almost $90 \%$ of ICC and ICC-related deaths occur in low/ middle-income countries (LMICs) due to a lack of resources and healthcare infrastructure needed to provide preventive services.

ICC and ICC-related mortality rates are particularly high in sub-Saharan Africa, which also has the highest rates of HIV infection in the world. Now, over 12 million HIV-infected (HIV-positive) women in sub-Saharan Africa are living longer because of antiretroviral therapy, thus increasing their likelihood of dying from ICC. ${ }^{3}$ However, many of these women are already exposed to human papillomavirus (HPV), the viral cause of ICC, and will not benefit from or be targeted for prophylactic HPV vaccination. Thus, 
cervical cancer screening will be needed for the foreseeable future.

However, setting up effective cytology for cervical cancer screening is expensive and requires a complex clinical and laboratory infrastructure that generally does not exist in LMICs. ${ }^{45}$ Moreover, it is now well understood that cytology has only a low to moderate one-time sensitivity for precursor lesions and therefore must be done repeatedly over many years to reduce cancer risk. Alternative strategies to address the ICG burden in LMICs, especially in SSA, must be developed and validated.

Persistent cervical infections by high-risk HPV (hrHPV) types cause virtually all ICC and its immediate precursor lesions, for example, cervical intraepithelial neoplasia grade 3 (CIN3) and adenocarcinoma in situ (AIS) everywhere in the world. ${ }^{67} \mathrm{hrHPV}$ causes most anal and vaginal cancers and a significant proportion of vulvar, penile and oropharyngeal cancers. ${ }^{8}$ HPV16 is the most important causal type, responsible for $\sim 60 \%$ of ICC. ${ }^{9}$ HPV1 8 is the next most important, responsible for $10 \%-15 \%$ of ICC, including $30 \%-40 \%$ of adenocarcinoma of the cervix, ${ }^{9}$ which is on the rise in Western countries. ${ }^{10}{ }^{11}$ Together, HPV16 and HPV18 account for $70 \%$ of ICC, and the same $15 \mathrm{hrHPV}$ types account for $99 \%$ of ICC everywhere in the world. ${ }^{9}$

There is now overwhelming evidence to suggest that testing for hrHPV is more sensitive, although less specific, than high-quality cytology for identifying women with cervical precancer. ${ }^{12-16}$ One-time hrHPV testing can reduce the risk of ICC incidence by approximately $40 \%$ in 6.5 years compared with cytology screening, ${ }^{16}$ and ICC mortality by approximately $40 \%$ (approximately $50 \%$ overall) in 8 years compared with cytology. ${ }^{17}$ Importantly, a negative hrHPV test provides superior reassurance against $\mathrm{CIN} 3+{ }^{18}$ and against ICC, ${ }^{16} 17$ permitting safe extension of screening intervals.

The WHO released cervical cancer screening and treatment guidelines in 2013, recommending two evidencebased approaches to cervical cancer screening ${ }^{19}$ : (1) Use either hrHPV testing or visual inspection after acetic acid (VIA), which involves the inspection of the cervix with a speculum in place and following the application of dilute acetic acid to help identify potential CIN by its characteristic white colouring in the presence of acetic acid (acetowhite), as alternative initial screening tests instead of Pap, and (2) immediately treat those who screen positive using the screening test, rather than require diagnostic verification through colposcopy and biopsy. This approach is commonly called screen-and-treat (S\&T), and is increasingly thought to be more amenable to LMIC settings.

However, hrHPV testing is also a much more effective screen than VIA ${ }^{17}$ which on a large scale appears to only downstage cancer rather than prevent it. ${ }^{20}$ Thus, the recent American Society for Clinical Oncology resource-stratified guidelines for secondary cervical cancer prevention $^{21} 22$ emphasise that hrHPV testing is the preferred choice for screening, with VIA only being used until hrHPV testing becomes available, and that HIV-infected women, because of their higher risk, should be screened twice as frequently as the general (HIV-uninfected) population.

Recent data in HIV-positive women living in the USA suggest that hrHPV testing may have clinical utility similar to that in HIV-negative women. Several observational studies have shown that an extended screening interval is safe in HIV-positive women who test hrHPV and Pap negative as it is for HIV-negative women. ${ }^{23}{ }^{24}$ In a study of women enroled in Women's Interagency Health Study in 2002, HIV-positive and HIV-negative women who tested hrHPV and Pap negative were at a similarly low risk of CIN2 or more severe (CIN2+) histology over a 5-year follow-up. ${ }^{23}$ In addition, no cases of histologically confirmed CIN2+ were diagnosed in the follow-up of hrHPV-negative and Pap-negative HIV-positive women aged 30-64 years who underwent routine 3-year hrHPV and cytology cotesting at Kaiser Permanente Northern California. ${ }^{24}$ Thus, both studies found very high negative predictive values (NPV) $>99 \%$ in HIV-positive women who test hrHPV negative. However, how hrHPV testing can best be used to screen HIV-positive women living in sub-Saharan Africa to prevent ICG remains to be determined.

Recent studies ${ }^{25-28}$ in HIV-positive women living in sub-Saharan Africa have compared hrHPV, VIA and/or Pap for the detection of cervical precancer/cancer. The results can be summarised as follows: (1) hrHPV detection was more sensitive but less specific than VIA; (2) cytology was equally or more sensitive but less specific than VIA; and (3) cytology was equally or more sensitive but less specific (vs the converse) than hrHPV testing. Results and conclusions are varied, leaving unanswered the question of what screening strategy in HIV-positive women living in sub-Saharan Africa has the greatest effectiveness and cost-effectiveness.

Regardless of the screening method, most screen-positive women who go to colposcopy or are treated immediately without diagnostic verification do not have cervical precancer and cancer (positive predictive value (PPV) for screening tests are typically $10 \%-20 \%$ ). In places like sub-Saharan Africa that lack necessary infrastructure and personnel such as pathologists, ${ }^{29}$ excessive referral to colposcopy is problematic. Although WHO recommendations for S\&T will hopefully overcome this bottleneck and increase the number of women living in LMICs who get screened, many countries may not adopt current S\&T strategies because of concerns of low specificity and overtreatment, resulting in increased costs, unnecessary patient discomfort and concern and wasting valuable healthcare resources that could otherwise be used to expand access to screening. Thus, methods to increase the accuracy of screening by reducing the numbers of women having colposcopy and biopsy or getting treated immediately in this context are highly desirable as they will likely increase the uptake of cervical cancer screening.

In order to improve the specificity of screening tests, secondary tests (biomarkers) are used following a 
screen-positive result with women who test positive for the triage undergoing further management (eg, colposcopy or immediate treatment) and those who test negative typically being deferred to further evaluation in 6-18 months to allow hrHPV infections to clear. There are several very promising biomarkers that might be used to improve the specificity and PPV of the screening tests. ${ }^{24}$ Given that HIV-infected women are more likely to test hrHPV positive, ${ }^{30-34}$ it is important to validate a triage strategy of using a secondary biomarker that sensitively and specifically rules in women with cervical precancer and cancer among the HIV-positive, hrHPV-positive women.

We are therefore conducting a cervical cancer screening study of $>5000$ Rwandan women, ages $30-54$ years, living with HIV infection. We are evaluating different screening tests (hrHPV DNA and VIA), those recommended by the WHO for cervical cancer screening, ${ }^{19}$ and different triage tests and biomarkers for the management of screen-positive women (E6/E7 oncoprotein detection, p16INK4a immunocytochemistry and hrHPV viral methylation). Screen-positive women undergo a rigorous colposcopic evaluation with multiple biopsies taken and the biopsies will undergo pathology review, to minimise the misclassification of endpoints. The primary objective of the study is to determine and compare clinical performance (Sensitivity (SE), Specificity (Sp), PPV and NPV) and cost-effectiveness for identifying HIV-positive women with CIN3+ and CIN2+ of different cervical cancer screening and management algorithms.

\section{METHODS AND ANALYSIS}

\section{Study design and population}

We are recruiting those women receiving care for confirmed HIV infection at health centres and various hospitals operated by the Ministry of Health or Rwanda Military Hospital (RMH) during 2016-2018 (table 1). Sites were selected in collaboration with Rwanda Biomedical Centre, which calculated estimated numbers of potentially eligible women using data from their HIV database (OpenMRS-Open Medical Records System; http:/ / openmrs.org/). The total of 7614 was the estimated number of women eligible for the study according to the inclusion/exclusion criteria (see below) at the beginning of the study in 2016. From each site, a convenience sample of women are being recruited to participate.

\section{Inclusion criteria}

Inclusion criteria include (1) living and receiving HIV care in Rwanda, (2) ages 30-54 years, (3) confirmed HIV positive based on medical records, (4) no prior cervical cancer screening, (5) no history of ICC and (6) willing, able and competent to provide written, informed consent. We are extending age range beyond that of the age range (30-49 years) recommended by the WHO for cervical cancer screening ${ }^{19}$ because there is limited evidence for the optimal upper age for cervical cancer screening of HIV-infected women.

\section{Exclusion criteria}

Exclusion criteria, in addition to not meeting the inclusion criteria, include (1) pregnant, (2) signs of abnormal, non-menstrual bleeding suggestive of ICC, (3) without a cervix due to hysterectomy and (4) not sufficiently healthy to participate in a research study based on the judgement of the clinicians. Excluded women are being advised to seek routine cervical cancer screening through government programmes.

Prior to initiation of enrolment at a specific clinic, the local HIV care provider team identifies potentially eligible women at their routine clinic visits and offers them enrolment. Women indicating interest in the study are then registered by our research nurses using the eligibility criteria checklist. All eligible and willing women at that site enrol and receive screening before moving to another site. The study team of at least two research nurses schedules 12 to 15 women 3 to 4 days a week by calling them and confirming appointments

Table 1 Recruitment sites and estimated eligible population

\begin{tabular}{llll}
\hline Province & Site & Type of site & $\begin{array}{l}\text { Potential participants per site } \\
\text { (approximate) }\end{array}$ \\
\hline Kigali & Cor-unum HC & Public Health Centre & 1405 \\
Kigali & Kimironko HC & Public Health Centre & 1227 \\
Kigali & Rwampara HC & Public Referral Hospital & 1098 \\
Kigali & Kicukiro HC & Public Health Centre & 1054 \\
Kigali & Kacyiru HC & Public Health Centre & 905 \\
Kigali & Gikondo HC & Public Health Centre & 925 \\
Kigali & Rwanda Military Hospital & Public Referral Hospital & 300 \\
Kigali & WEACTx for Hope & Private HIV Clinic & 500 \\
Kigali & Busanza HC & Public Health Centre & 100 \\
Kigali & Nyarugunga HC & Public Health Centre & 100 \\
Total & & & 7614 \\
\hline
\end{tabular}




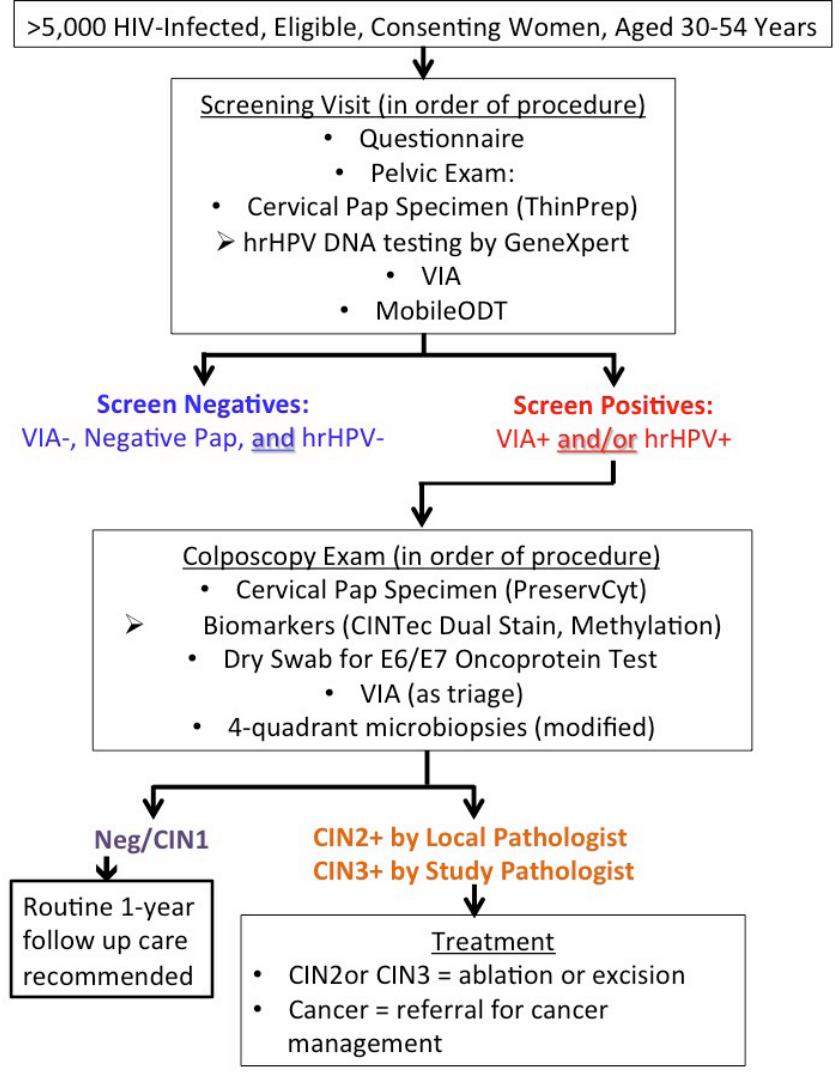

Figure 1 Study design. CIN2/CIN3, cervical intraepithelial neoplasia grade 2/cervical intraepithelial neoplasia grade 3; hrHPV, high-risk HPV; VIA, visual inspection after acetic acid.

over the telephone. Two teams of nurses are in the field, meaning that two cervical cancer screening clinics can be run simultaneously.

\section{Enrolment visit}

The study participant flow is summarised in figure 1 . Enrolment visits, including pelvic examinations with VIA and specimen collection, are being done entirely by a team of two study nurses. During their enrolment visit, women are being educated on cervical cancer risk factors, mainly HPV infection, and why they are more at risk to develop ICC than HIV-uninfected women. They also are being allowed to ask questions before they commit to participating in the study. Women are then being asked to provide informed, written consent to participate in the study using a printed consent form. Those who provide consent complete a short nurse-administered questionnaire (see online supplementary appendix I) on cervical cancer risk factors and sociodemographic characteristics using a data capture screen in Microsoft Access. The questionnaire collects information on basic sociodemographics, factors associated with acquiring HPV (eg, marital status and recent and lifetime number of sexual partners), factors associated with increased risk of progression of hrHPV infection to precancer and cancer (eg, smoking and other tobacco use, parity and oral and other contraceptive use) and other infections common in Rwanda such as malaria ${ }^{35}$ and tuberculosis ${ }^{36}$ that have been previously reported to be associated with precancer among hrHPV-infected women. The questionnaire was not pretested.

Enroled women then undergo a pelvic examination, with VIA and a single cervical exfoliated ('Pap') specimen collected and placed into $20 \mathrm{~mL}$ PreservCyt (Hologic, Bedford, Massachusetts, USA) which is then being sent to the laboratory at RMH for hrHPV testing. Finally, a portable colposcope (http://www.mobileodt.com/; MobleODT, Tel Aviv, Israel) is being used for digital cervicography (comparable to VIA with magnification) and the image is being captured and saved for quality control and research, and to develop a digital library.

\section{Colposcopy visit}

Screen-positive women (women who test hrHPV and/or VIA positive) are being called using a telephone as soon as the hrHPV result is available and being invited to return for colposcopy within 1 month. All screen-positive women receiving colposcopy will be having two additional specimens collected, one into PreservCyt for the evaluation of other molecular biomarkers (genotype-specific hrHPV viral methylation and load, and p16/Ki-67 immunocytochemistry CINtec PLUS Cytology Kit (Roche, Tucson, Arizona, USA) ) and a second as a dry swab for HPV16, 18, $31,33,35,45,52$ and $58 \mathrm{E} 6 / \mathrm{E} 7$ oncoprotein detection by the next-generation lateral flow hrHPV oncoprotein test from Arbor Vita Corporation (Fremont, California, USA) being included in this study as a triage for screen-positive women to identify those women who are at higher risk of having CIN3+. The residual PreservCyt specimens from both the screening and colposcopy visits are being stored at $-20^{\circ} \mathrm{C}$, creating a biobank in Rwanda for future retrospective evaluations of promising new biomarkers and tests.

After specimen collection, a colposcopic evaluation of the cervix is being done with a modified version of the four-quadrant microbiopsy procedure being performed. ${ }^{37}$ Compared with the standard biopsy, the microbiopsy protocol improves disease ascertainment and reduces biases related to selecting the most visually obvious acetowhite lesions while removing less tissue $\left(\sim 13 \mathrm{~mm}^{2}\right.$ for four microbiopsies vs $\sim 28 \mathrm{~mm}^{2}$ for one standard biopsy). Modifications to the standard four-quadrant microbiopsy procedure are: (1) endocervical curettage is being taken only for those women whose squamocolumnar junction is not entirely visible and the lesion extends into the endocervical canal; and (2) standard-size biopsies of very large lesions are being taken to increase the likelihood that the most severe area is being biopsied.

\section{Pathology}

Biopsies are being processed in a single cassette so that a single slide has a section from all biopsies taken. Biopsies are being read by a local pathologist at RMH and Dr Hebert or another pathologist at Montefiore Medical 
Centre, Bronx, New York, USA. Women receiving a diagnosis of CIN2+ by the Rwandan pathologist (TZ) or, as a safety precaution, CIN3+ diagnosis by Montefiore pathologist (TH) are receiving treatment ${ }^{38}$ : (1) CIN2, CIN3 or AIS are being referred to study doctors to undergo an excision procedure (eg, loop electrosurgical excision procedure (LEEP) or cold-knife cone (CKC)) and (2) ICC are being referred to RMH Hospital for care. Women with $<$ CIN2 are being advised to seek rescreening in a year.

A slide with biopsies is undergoing p16 immunohistochemistry (IHC) using the CINtec Histology Kit (Roche) for research purposes only.

\section{Endpoints}

The primary scientific endpoints of the study are histologically confirmed, consensus CIN2+, that is, both pathologists diagnose CIN2+ (without adjudication) or CIN3+ by the study pathologist. The secondary, clinical endpoint is histologically confirmed CIN2+ diagnosed by the Rwandan pathologist. Additional endpoints using pathology review and p16 IHC are being used but not for evaluating the performance of p16 immunocytochemistry due to the possibility of p16-related autocorrelation.

\section{Treatment}

Women diagnosed with CIN2+ are being referred for treatment. Those precancerous lesions are being treated by ablation if they meet WHO criteria for cryotherapy. ${ }^{39}$ Those who do not meet those criteria undergo an excision procedure (eg, LEEP or CKC) or, in the case of an ICC diagnosis, referred for cancer management. Screen-positive women with $<$ CIN2 are being advised to seek rescreening in a year through the existing healthcare system.

\section{Data sources}

Data are being collected from the following sources:

1. A nurse-administered questionnaire on sociodemographic characteristics and cervical cancer risk factors including age at first sexual intercourse, number of sexual partners, smoking, contraception, parity and socioeconomic status.

2. Pelvic examination, VIA, MobileODT and colposcopy data capture forms.

3. Medical record data on HIV status (eg, (http://www. who.int/hiv/pub/guidelines/HIVstaging150307. pdf), CD4 count, viral load, antiretroviral therapy regimen(s), care and dates.

\section{Laboratory testing}

The following laboratory tests are being performed:

\section{Xpert HPV testing}

Cervical Pap specimens in PreservCyt are being sent to the RMH laboratory in Kigali, Rwanda for hrHPV DNA testing using the Xpert HPV test (Cepheid, Sunnyvale, California, USA). ${ }^{40-45}$ The Xpert HPV assay is a new, qualitative, real-time PCR assay for the detection of hrHPV
DNA. The Xpert HPV assay includes simultaneous detection of 14 hrHPV types, hydroxymethylbilane synthase (HMBS) and an internal Probe Check Control. The 14 targeted hrHPV types are detected in five fluorescent channels: (1) HPV16, (2) HPV18 and hrHPV 45 (HPV18/45), (3) HPV31, 33, 35, 52 and 58, (4) HPV51 and HPV59 and (5) HPV39, 56, 66 and 68. HMBS (fluorescent channel 6) verifies specimen adequacy.

Specimens are being mixed and a $1 \mathrm{~mL}$ prealiquot is being removed using a disposable pipette and placed in the testing cartridge per the manufacturer's instructions. Unsatisfactory results due to insufficient cellular content are being retested. If the second test is also unsatisfactory, the final result is being recorded as unsatisfactory but women with unsatisfactory results are being referred to colposcopy for safety.

\section{HPV viral methylation}

We will conduct a retrospective analysis of HPV viral methylation and its association with CIN2+. To identify single hrHPV-type infections, we will select single-channel positives from the Xpert HPV assay. For those that are hrHPV positive for a channel other than HPV16, which is detected singly, we will test them to identify the single-type infections using a standard protocol for PCR amplification using MY09/11 L1 consensus primers and hrHPV genotype detection using dot hybridisation for 39 individual type-specific probes and a mixture of probes for 10 other uncommon hrHPV types as previously described. ${ }^{46} 47$ To isolate the DNA, ThinPrep specimens $(1.5 \mathrm{~mL})$ will be pelleted, resuspended in Sucrose, Tris and Magnesium Chloride (STM), digested with Proteinase K, precipitated overnight in ammonium acetate ethanol at $-20^{\circ} \mathrm{C}$, washed and suspended and stored in Tris EDTA (TE) buffer.

The isolated DNA will then undergo bisulfite conversion. ${ }^{48}$ Following bisulfite conversion and DNA purification and desulfonation, bisulfite-treated DNA will be used as template for Next-Gen Sequencing (NGS) (HiSeq2000, Illumina, San Diego, California, USA) using barcoded-type specific primers. Sequences for pads and barcodes are not found in the targeted genomic region. Use of padding and barcodes will enable reads to be identified by amplicon (forward or reverse) or by sample during downstream bioinformatics analysis. ${ }^{49}$

All PCR products for NGS will be pooled (by assay) and a single DNA band containing multiple amplicons from different samples (with unique barcodes) will be isolated from a gel for NGS. ${ }^{49}$ Briefly, equal concentrations of each barcoded PCR product (based on PCR band intensity) will be pooled and isolated. On confirmation of correct product size, all purified DNA pools will be combined and submitted for library preparation and paired-end 100 base pair Illumina HiSeq2000 sequencing at the Einstein Genomics Core Facility.

Methylation status are being determined in the laboratory of Dr Robert Burk at Albert Einstein College of Medicine (USA). Prior to determination of methylation status, demultiplexing based on the unique barcodes 
is being performed using in-house generated scripts to obtain paired-end NGS reads of each sample. Reads are being aligned with hrHPV reference genome sequences by bowtie v0.12.9. ${ }^{50}$ Methylation status of each CpG site is then determined by bismark v0.7.7 $7^{51}$ using the default quality score parameter set to Q30, and the formula of the methylation ratio of the number of $\mathrm{C}$ read by the number of $\mathrm{C}+\mathrm{T}$ read.

\section{E6/E7 oncoprotein testing}

Dry swab specimens, collected at the time of colposcopy, are being tested for individual E6/E7 oncoproteins as previously described, ${ }^{5253}$ according to the manufacturer's instructions, at the RMH laboratory in Kigali, Rwanda. The E6/E7 oncoprotein test uses three lateral flow strips to detect eight hrHPV types whereas the E6 oncoprotein test used a single lateral flow strip to detect three hrHPV types.

\section{Analyses}

We will evaluate combinations of the above-mentioned screening strategies and tests to estimate the clinical performance (eg, SE, Sp, PPV and NPV) for the detection of consensus CIN3+ and community CIN2+. A log binomial model using generalised estimating equations will be used to take into account correlation between different tests from the same subject. Note that while these models will be developed for the estimation and comparison of performance for two tests, the model can be extended to allow more than two tests by including more indicator variables for test type.

Some analyses of biomarkers such as viral methylation will be restricted to hrHPV positives. Comparisons of hrHPV viral methylation to other triage biomarkers will be restricted to the subset that gets tested for viral methylation as described.

\section{Sample size calculations}

We are basing our sample size on the ability to detect modest but minimally important differences in Se of $15 \%$. We conservatively assume that the population prevalence of CIN3+ is $2 \%$ based on our past study in Rwanda. ${ }^{335}$ We will enrol and have complete follow-up of at least 5000 HIV-positive women. A sample size of 5000 HIV-positive women with completed follow-up of the screen positives will yield 100 cases of CIN3+, which will have at least $80 \%$ power $(\alpha=5 \%)$ to crudely detect a $15 \%$ difference in Se between two screening strategies for a range of 10\%-25\% discordance. With this sample size of 5000 women, 4900 will not have CIN3+; we will also have at least $90 \%$ power $(\alpha=5 \%)$ to detect a difference in Sp of $3 \%$ for discordance up to $40 \%$. Finally, we will have $80 \%$ power $(\alpha=5 \%)$ to crudely detect an $8 \%, 10 \%$ or $11 \%$ difference in PPV if the reference PPV is $10 \%, 20 \%$ or $30 \%$, respectively. ${ }^{54}$

\section{Cost-effectiveness}

We will conduct assessments of the costs and cost-effectiveness of the different combinations of screening and triage tests, that is, algorithms, as well as those of the entire community-based screening 'system'. Costs measurement will be conducted using a microcosting (ingredients) approach in which resource use throughout each step in the screening process will be tracked and unit costs for each of the resources will be applied to generate an average screening cost per woman to be compared against what the estimated costs are for a possible programme based on hrHPV screening and VIA triage or VIA screening. For estimating costs of the screening system and scale-up of screening to 100000 women in a month, analyses will distinguish financial costs, which reflect actual expenditures of the programme, from economic costs, including the value of donated and shared resources to more fully assess opportunity costs. Projections on budget impact and economic cost implications over time will be made under varying assumptions of screening uptake, follow-up compliance and scenarios of changing disease burden.

Clinical outcomes will include true positive, true negative, false negative and false positive test results, number of colposcopies, incident cancer and cancer death. Cost-effectiveness will be measured as cost/CIN2+ detected, cost/CIN3+ detected, cost/invasive cancer prevented, cost/cancer death prevented, cost/life-year saved and cost/quality-adjusted life year (QALY) saved; in addition, we will calculate harm:benefit ratios, using varying definitions of harms (colposcopies, false positive results) to benefits (cancers prevented, deaths prevented, lifeyears and QALYs saved). Costs and effectiveness will be discounted at a $3 \%$ annual rate, with the rate varied from $0 \%$ to $5 \%$ in sensitivity analysis. For assessment of value of information, we will use net monetary benefits (NMB), defined as a function of the willingness-to-pay (WTP) threshold for different costs and outcomes as: $\mathrm{NMB}=($ WTP $*$ Effectiveness $)-$ Costs.

\section{PATIENT AND PUBLIC INVOLVEMENT}

There was no patient engagement in the development or design of the study, recruitment and the conduct of the study. Participants are receiving their results directly since it is related to their care. As this was not a randomised controlled trial, the burden of the intervention was not assessed by patients themselves. There were no patient advisors to acknowledge.

\section{ETHICS AND DISSEMINATION \\ Ethics}

This study protocol was reviewed and approved.

\section{Confidentiality measures and protection against potential risks}

The risks for those participating in our study include:

- Collection of Pap specimens/cervical swabs involves a modest risk of bleeding which is typically very limited when it occurs. Testing positive for any test may cause psychological distress (anxiety). 
- Colposcopy and excisional treatments induce vaginal bleeding and may incur pain, infection and short-term psychological distress (anxiety). A diagnosis of CIN2 or CIN2+ may cause psychological distress (anxiety). A diagnosis of ICC may cause severe psychological distress.

- Questions in the questionnaire, regarding sexual behaviour and other matters of a personal nature, may cause anxiety and embarrassment. Participants are advised that they are free to not answer specific questions.

- There is also the risk of psychosocial stress which could occur if there was inadvertent disclosure of confidential medical or other personal information.

Protection against the risk of inadvertent disclosure of confidential information is being addressed by the standard procedures at the Rwandan study site: (1) storing completed paper copies of questionnaires and other hard copy information (described above), identified by study number only, in a filing system separate from the name-address file of participants in the study; (2) only the designated local personnel have access to cross-reference the files; (3) all paper files, including consent forms, are being maintained in locked cabinets in locked rooms, with access restricted to specific research personnel.

In addition, we will include the following security measures to protect the data:

- Controlled access to project data;

- A tracking system for data forms and activities;

- Date and time of stamping of all data records with electronic signatures;

- Audit trails to track all changes made to data records; and

- Data kept on password-protected computers and in locked rooms.

\section{Potential patient benefits}

There are potential direct benefits to study participants. HIV-positive women participating in the study are at very high risk of ICC. They are being rigorously screened and evaluated, more effectively than the standard of care anywhere in the world. As a result of the study, women with precancer who are at imminent risk of ICC are being diagnosed sooner and treated more effectively than women receiving routine care and thereby more likely averting the development of ICC. Women with cervical cancer are being diagnosed earlier thereby reducing the morbidity and the risk of mortality caused by ICC. Conversely, any pain, bleeding or stress that might occur related to colposcopy or cervical swab are typically modest and well tolerated.

There are also substantial potential societal benefits related to the study due to its implications to improving cervical cancer screening practices and guidelines in HIV-positive women-changes in practice which might also benefit the study participants themselves, if and when these changes are enacted. There is a great need to identify more effective and practical methods for cervical cancer screening for HIV-positive women living in Africa, who are living longer than ever and are therefore at potentially greater risk of ICC.

\section{Dissemination}

We plan to publish a series of scientific reports in peer-reviewed scientific journals. As building research capacity in Rwanda is a major goal of this research project, all investigators of the research team are being asked and supported to lead at least one analysis and one manuscript preparation, based on interests and expertise.

In addition, an external advisory panel (EAP) composed of leaders from the Rwanda Ministry of Health, University of Rwanda and Rwanda medical community has been formed. The responsibilities of the EAP include providing advice on the conduct of the project and interpretation for and dissemination of the study results to Rwandan stakeholders. The latter is important for the adoption of evidence-based best practices for cervical cancer screening as warranted.

\section{LIMITATIONS}

There are several limitations to the study that bear mentioning. First, cervical cytology is not being included in the study. There is limited cervical cytology services available locally and of unknown quality and it is unlikely that cytology will be widely available in Rwanda, making its inclusion as a comparator test of limited value. Moreover, there are significant costs and logistical challenges in shipping PreservCyt specimens to and having cytology slides made and read in the USA. Second, we are not conducting colposcopy and taking biopsies in screen-negative women, which would have allowed us to estimate absolute clinical performance. The burden of sending screen-negative women to colposcopy is deemed too great and it is impractical to send a sufficient number of screen-negative women to colposcopy to accurately estimate the false negative disease (CIN3+) fraction. Thus, only relative clinical performance of the screening tests will be estimated from this study.

Contributors KA, PEC, JCD, AA and JDS conceived the original concept of the study and the interventions. GM, KA and PEC drafted the protocol. PEC performed the sample size calculations, and PEC and GM will lead analysis of the results. GM, JDS and AM are supporting patient recruitment. GM, TR and AM are providing clinical care for patients. AM and LM oversee laboratory testing, and TZ and TH are responsible for pathology. GM and LM oversee and administer the study activities at the clinical site in Rwanda. All authors contributed to the scientific design of the study and the protocol development, are involved in the implementation of the project and have read and approved the final manuscript.

Funding This study was funded by NCI/NIH Grant 5U54CA19016304 and by a grant from the Prevent Cancer Foundation.

Competing interests This research study has received HPV tests for reduced or no cost from Cepheid, Arbor Vita Corporation, and Roche.

Patient consent Obtained.

Ethics approval Institutional Review Board for human subjects research at Albert Einstein College of Medicine and Rwanda National Ethics Committee.

Provenance and peer review Not commissioned; externally peer reviewed. 
Open access This is an open access article distributed in accordance with the Creative Commons Attribution Non Commercial (CC BY-NC 4.0) license, which permits others to distribute, remix, adapt, build upon this work non-commercially, and license their derivative works on different terms, provided the original work is properly cited, appropriate credit is given, any changes made indicated, and the use is non-commercial. See: http://creativecommons.org/licenses/by-nc/4.0/.

\section{REFERENCES}

1. Ferlay J, Soerjomataram I, Ervik M, et al. GLOBOCAN 2012 v1.0, Cancer incidence and mortality worldwide: IARC CancerBase No. 11. Lyon, France: International Agency for Research on Cancer, 2013.

2. Lönnberg S, Hansen BT, Haldorsen T, et al. Cervical cancer prevented by screening: Long-term incidence trends by morphology in Norway. Int J Cancer 2015;137:1758-64.

3. UNAIDS. UNAIDS Data. 2017

4. Cervix Cancer Screening. IARC Handbooks of Cancer Prevention: IARC Press, 2005

5. Kitchener HC, Castle PE, Cox JT. Chapter 7: achievements and limitations of cervical cytology screening. Vaccine 2006;24:S63-70.

6. Schiffman M, Castle PE, Jeronimo J, et al. Human papillomavirus and cervical cancer. The Lancet 2007;370:890-907.

7. Schiffman M, Clifford G, Buonaguro FM. Classification of weakly carcinogenic human papillomavirus types: addressing the limits of epidemiology at the borderline. Infect Agent Cancer 2009;4:8.

8. Forman D, de Martel C, Lacey CJ, et al. Global burden of human papillomavirus and related diseases. Vaccine 2012;30(Suppl 5):F12-23.

9. SS de, Quint WG, Alemany L, et al. Human papillomavirus genotype attribution in invasive cervical cancer: a retrospective cross-sectional worldwide study. Lancet Oncol 2010;11:1048-56.

10. Bray F, et al. Incidence trends of adenocarcinoma of the cervix in 13 European Countries. Cancer Epidemiol Biomarkers Prev 2005:14:2191-9.

11. Adegoke $O$, Kulasingam S, Virnig B. Cervical cancer trends in the united states: a 35-year population-based analysis. J Womens Health 2012;21:1031-7.

12. Cuzick J, Clavel C, Petry K-U, et al. Overview of the European and North American studies on HPV testing in primary cervical cancer screening. Int J Cancer 2006;119:1095-101.

13. Naucler P, Ryd W, Törnberg S, et al. Human papillomavirus and papanicolaou tests to screen for cervical cancer. N Engl J Med Overseas Ed 2007;357:1589-97.

14. Ronco G, Giorgi-Rossi P, Carozzi F, et al. Efficacy of human papillomavirus testing for the detection of invasive cervical cancers and cervical intraepithelial neoplasia: a randomised controlled trial. Lancet Oncol 2010.

15. Rijkaart DC, Berkhof J, Rozendaal L, et al. Human papillomavirus testing for the detection of high-grade cervical intraepithelial neoplasia and cancer: final results of the POBASCAM randomised controlled trial. Lancet Oncol 2012;13:78-88.

16. Ronco G, Dillner J, Elfstrom KM, et al. Efficacy of HPV-based screening for prevention of invasive cervical cancer: follow-up of four European randomised controlled trials. Lancet 2013.

17. Sankaranarayanan R, Nene BM, Shastri SS, et al. HPV Screening for cervical cancer in rural India. N Engl J Med 2009;360:1385-94.

18. Dillner J, Rebolj M, Birembaut P, et al. Long term predictive values of cytology and human papillomavirus testing in cervical cancer screening: joint European cohort study. BMJ 2008;337:a1754

19. World Health Organization. New guidelines on screening and treatment for cervical cancer. South Africa: World Health Organization, 2013.

20. Shastri SS, Mittra I, Mishra GA, et al. Effect of VIA Screening by primary health workers: randomized controlled study in Mumbai, India. J Natl Cancer Inst 2014;106:dju009.

21. Jeronimo J, Castle PE, Temin S, et al. Secondary prevention of cervical cancer: american society of clinical oncology resourcestratified clinical practice guideline summary. J Oncol Pract 2017;13.

22. Castle PE, Jeronimo J, Temin S, et al. Screening to prevent invasive cervical cancer: ASCO resource-stratified clinical practice guideline. $J$ Clin Oncol 2017;35:1250-2.

23. Keller MJ, Burk RD, Xie X, et al. Risk of cervical precancer and cancer among HIV-infected women with normal cervical cytology and no evidence of Oncogenic HPV Infection. JAMA 2012;308:362-9.

24. Castle PE, Fetterman B, Poitras N, et al. Safety against cervical precancer and cancer following negative human papillomavirus and papanicolaou test results in human immunodeficiency virus-infected women. Arch Intern Med 2012;172:1041-3.
25. Chung MH, McKenzie KP, Vh D, et al. Comparing pap smear, via, and hpv cervical cancer screening methods among hiv-positive women by immune status and antiretroviral therapy. AIDS 2013.

26. Firnhaber C, Mayisela N, Mao L, et al. Validation of cervical cancer screening methods in hiv positive women from Johannesburg South Africa. PLoS One 2013;8:e53494.

27. Dartell MA, Rasch V, Iftner T, et al. Performance of visual inspection with acetic acid and human papillomavirus testing for detection of high-grade cervical lesions in HIV positive and HIV negative Tanzanian women. Int J Cancer 2014;10.

28. Mabeya H, Khozaim K, Liu T, et al. Comparison of conventional cervical cytology versus visual inspection with acetic acid among human immunodeficiency virus-infected women in Western Kenya. $J$ Low Genit Tract Dis 2012;16:92-7.

29. Adesina A, Chumba D, Nelson AM, et al. Improvement of pathology in sub-Saharan Africa. Lancet Oncol 2013;14:e152-e157.

30. Dartell M, Rasch V, Kahesa C, et al. Human papillomavirus prevalence and type distribution in 3603 hiv-positive and hivnegative women in the general population of Tanzania. Sex Transm Dis 2012;39:201-8.

31. D'Souza G, Burk RD, Zhong Y, et al. Cervicovaginal human papillomavirus (HPV)-infection before and after hysterectomy: evidence of different tissue tropism for oncogenic and nononcogenic HPV types in a cohort of HIV-positive and HIV-negative women. Int $J$ Cancer 2012;131:1472-8.

32. Ng'andwe C, Lowe JJ, Richards PJ, et al. The distribution of sexually-transmitted Human Papillomaviruses in HIV positive and negative patients in Zambia, Africa. BMC Infect Dis 2007;7:77.

33. Singh DK, Anastos K, Hoover DR, et al. Human papillomavirus infection and cervical cytology in hiv-infected and hiv-uninfected rwandan women. J Infect Dis 2009;199:1851-61.

34. Marais DJ, Vardas E, Ramjee G, et al. The impact of human immunodeficiency virus type 1 status on human papillomavirus (hpv) prevalence and HPV Antibodies in serum and cervical secretions. $J$ Infect Dis 2000;182:1239-42.

35. Anastos K, Hoover DR, Burk RD, et al. Risk factors for cervical precancer and cancer in HIV-infected, HPV-positive rwandan women. PLoS One 2010;5:e13525.

36. Zhao FH, Varanasi AP, Cunningham CA, et al. Tuberculosis and oncogenic HPV: potential co-infections in women at highrisk of cervical cancer in rural China. Asian Pac J Cancer Prev 2011;12:1409-15.

37. Pretorius RG, Zhang W-H, Belinson JL, et al. Colposcopically directed biopsy, random cervical biopsy, and endocervical curettage in the diagnosis of cervical intraepithelial neoplasia II or worse. Am J Obstet Gynecol 2004;191:430-4.

38. Schiffman M, Adrianza ME. Design, methods and characteristics of trial participants. Acta Cytol 2000;44:726-42.

39. World Health Organization. WHO guidelines for treatment of cervical intraepithelial neoplasia 2-3 and adenocarcinoma in situ: World Health Organization, 2014.

40. Castle PE, Smith KM, Davis TE, et al. Reliability of the Xpert HPV assay to detect high-risk human papillomavirus dna in a colposcopy referral population. Am J Clin Pathol 2015;143:126-33.

41. Cuzick J, Cuschieri K, Denton K, et al. Performance of the Xpert HPV assay in women attending for cervical screening. Papillomavirus Res 2015;1:32-7.

42. Einstein MH, Smith KM, Davis TE, et al. Clinical evaluation of the cartridge-based genexpert human papillomavirus assay in women referred for colposcopy. J Clin Microbiol 2014.

43. Toliman P, Badman SG, Gabuzzi J, et al. Field evaluation of xpert hpv point-of-care test for detection of human papillomavirus infection by use of self-collected vaginal and clinician-collected cervical specimens. J Clin Microbiol 2016;54:1734-7.

44. Cuschieri K, Geraets D, Cuzick J, et al. Performance of a cartridge-based assay for detection of clinically significant Human Papillomavirus (HPV) Infection: lessons from VALGENT (Validation of HPV Genotyping Tests). J Clin Microbiol 2016;54:2337-42.

45. Kunckler M, Schumacher F, Kenfack B, et al. Cervical cancer screening in a low-resource setting: a pilot study on an HPV-based screen-and-treat approach. Cancer Med 2017;10.

46. Guimarães MDC, Grinsztejn B, Melo VH, et al. Anal HPV prevalence and associated factors among hiv-seropositive men under antiretroviral treatment in Brazil. J Acquir Immune Defic Syndr 2011;57(Suppl 3):S217-S224.

47. Castle PE, Schiffman M, Gravitt PE, et al. Comparisons of HPV DNA detection by MY09/11 PCR methods. J Med Virol 2002;68:417-23.

48. Hayatsu $\mathrm{H}$, Shiraishi M, Negishi K. Bisulfite modification for analysis of DNA methylation. Curr Protoc Nucleic Acid Chem 2008. 
49. Smith BC, McAndrew T, Chen Z, et al. The cervical microbiome over 7 years and a comparison of methodologies for its characterization. PLoS One 2012;7:e40425

50. Langmead B, Trapnell C, Pop M, et al. Ultrafast and memory-efficient alignment of short DNA sequences to the human genome. Genome Biol 2009;10:R25.

51. Krueger F, Andrews SR. Bismark: a flexible aligner and methylation caller for bisulfite-seq applications. Bioinformatics 2011;27:1571-2.
52. Qiao YL, Jeronimo J, Zhao FH, et al. Lower cost strategies for triage of human papillomavirus DNA-positive women. Int J Cancer 2013.

53. Zhao F-H, Jeronimo J, Qiao Y-L, et al. An evaluation of novel, lowercost molecular screening tests for human papillomavirus in rural China. Cancer Prev Res 2013;6:938-48.

54. Leisenring W, Alono T, Pepe MS. Comparisons of predictive values of binary medical diagnostic tests for paired designs. Biometrics 2000;56:345-51. 